Pacific Journal of Mathematics

ASYMPTOTIC VALUES OF A HOLOMORPHIC FUNCTION 


\title{
ASYMPTOTIC VALUES OF A HOLOMORPHIC FUNCTION WITH RESPECT TO ITS MAXIMUM TERM
}

\author{
Alfred Gray and S. M. Shah
}

Let $f(z)=\sum_{n=0}^{\infty} a_{n} z^{n}$ be holomorphic with radius of convergence $R(0<R \leqq \infty)$, and let $\mu(r)$ denote the maximum term and $\nu(r)$ the central index of $f(z)$. By definition for $r>0, \mu(r)=\max \left\{\left|a_{n}\right| r^{n} \mid n=0,1,2, \cdots\right\}$ and $\nu(r)=\max \{n \mid \mu(r)=$ $\left.\left|a_{n}\right| r^{n}\right\}$ so that $\mu(r)=\left|a_{\nu(r)}\right| r^{\nu(r)}$. In previous papers we have investigated the limiting values of the quotient $\mu(r) / M(r)$ as $r \rightarrow R$. Here, as usual, $M(r)$ denotes the maximum modulus of $f(z)$. Recently Clunie and Hayman have disproved a conjecture of Erdös that if $\mu(r) / M(r)$ tends to a limit, the limit must be zero.

In this paper we consider a more general problem. There are two complex functions $\mu(z)$ and $m(z)$ which can be regarded as complex extensions of $\mu(r)$ in a natural way. We are led to investigate the limiting values of $f(z) / \mu(z)$ and $f(z) / m(z)$ along curves tending to $|z|=R$, and we call these $\mu$ and $m$ asymptotic values. We prove that for a class of functions which are either of very slow growth, or have gap power series, there are no $\mu$ or $m$ asymptotic values. On the other hand, for the admissible functions of Hayman, $\infty$ is a $\mu$ and $m$ asymptotic value along the positive real axis, while 0 is a $\mu$ and $m$ asymptotic value along any other path in an angle excluding the positive real axis.

Definitions. First we extend $\mu$ to a complex function by the formula

$$
\mu\left(r e^{i \theta}\right)=\mu(r) e^{i \nu(r) \theta},
$$

for $r>0$ and $0 \leqq \theta<2 \pi$. Then $\mu(r)=\left|\mu\left(r e^{i \theta}\right)\right|$ and $\mu(z)=\left|a_{\nu(|z|)}\right| z^{\nu(|z|)}$. We also define a "complex maximum term" $m(z)$ given by

$$
m\left(r e^{i \theta}\right)=\mu(r) \exp \left\{i \nu(r) \theta+i \arg a_{\nu(r)}\right\}
$$

for $r>0$ and $0 \leqq \theta<2 \pi$. Then $m(z)=a_{\nu(|z|)} \nu^{\nu(|z|)}$ and as before $\mu(r)=$ $\left|m\left(r e^{i \theta}\right)\right|$. Note that $\mu(z)$ and $m(z)$ are continuous in each annulus where $\nu(|z|)$ is continuous, but in general have discontinuities where $\nu(|z|)$ is discontinuous.

Let $\gamma(t)$ be a (continuous) curve such that $|\gamma(t)| \rightarrow R$ as $t \rightarrow \infty$. If $f(\gamma(t)) / \mu(\gamma(t))(f(\gamma(t)) / m(\gamma(t)))$ tends to a limit $\omega(0 \leqq|\omega| \leqq \infty)$ as $t \rightarrow \infty$ we say that $\omega$ is a $\mu$ asymptotic value ( $m$ asymptotic value) of $f(z)$ and $\gamma(t)$ is a corresponding $\mu$ asymptotic path ( $m$ asymptotic 
path). Further let $\gamma(t)$ be a $\mu($ or $m$ ) asymptotic path written in polar coordinates $(r(t), \theta(t))$. Then $\gamma(t)$ is nonessential if and only if there exists $\varepsilon>0$ such that for all curves of the form $(r(t), \phi(t))$ such that $|\phi(t)-\theta(t)|<\varepsilon$ for sufficiently large $t$ we have that $(r(t), \theta(t))$ is a $\mu($ or $m$ ) asymptotic path with the same $\mu$ (or $m$ ) asymptotic value as $(r(t), \theta(t))$. Otherwise $\gamma(t)$ is essential. Note that $f$ has $\mu($ or $m)$ asymptotic value $\infty(0)$ if and only if $|f(z)| / \mu(|z|) \rightarrow \infty(0)$ along a curve $\gamma(t)$. Also if $a_{n} \geqq 0$ for sufficiently large $n$ then the $\mu$ and $m$ asymptotic values are the same, as are the $\mu$ and $m$ asymptotic paths.

Let $\{\rho(n)\}$ be the sequence of jump points of $\nu(r)$, counting multiplicity, and assume throughout this paper that $\mu(r) \rightarrow \infty$ as $r \rightarrow R$ (so that $\rho(n) \rightarrow R$ as $n \rightarrow \infty$ ). This last assumption avoids triviality and implies that if $a_{n} \geqq 0$ for $n>n_{0}$ then $\mu(z)=m(z)$ for $|z|$ sufficiently near $R$. We denote by $\left\{n_{k}\right\}$ the range of $\nu(r)$ (so that $\left.\nu\left(\rho\left(n_{k}\right)\right)=n_{k}\right)$ and we define:

$$
\begin{aligned}
& L=\limsup _{k \rightarrow \infty} \rho\left(n_{k+1}\right) / \rho\left(n_{k}\right) . \\
& S=\limsup _{k \rightarrow \infty}\left(n_{k+1}-n_{k}\right) \text {. } \\
& \left.\begin{array}{l}
A \\
\mathrm{a}
\end{array}\right\}=\lim _{k \rightarrow \infty \sup } \frac{n_{k+1}-n_{k}}{n_{k}-n_{k-1}} \text {. } \\
& \left.\begin{array}{l}
\Phi \\
\phi
\end{array}\right\}=\limsup _{k \rightarrow \infty \inf }\left(n_{k+1}-n_{k}\right) \log \left(\frac{\rho\left(n_{k+1}\right)}{\rho\left(n_{k}\right)}\right) \text {. }
\end{aligned}
$$

THeOREM 1. If $L>1$ and $S<\infty$, then $f(z)$ has no $\mu$ or $m$ asymptotic values. (The hypothesis $L>1$ implies $f(z)$ is a transcendental entire function.)

\section{Statement of theorems.}

Theorem 2. Suppose $0<\phi=\Phi<\infty$ and $1 \leqq \alpha=A<\infty$, and that $f(z)$ has the form

$$
f(z)=1+\sum_{k=1}^{\infty} \frac{z^{n_{k}}}{\rho(1) \cdots \rho\left(n_{k}\right)} .
$$

Then $f(z)$ has no $\mu$ asymptotic values.

Next suppose that $f(z)$ is real for real $z$ and $f(r)>0$ for $R_{0}<r<R$. Let

$$
a(r)=\frac{r f^{\prime}(r)}{f(r)} \quad \text { and } \quad b(r)=r a^{\prime}(r) .
$$

Following Hayman [9] we call $f(z)$ admissible if $b(r) \rightarrow+\infty$ as $r \rightarrow R$ 
and there exists a function $\delta(r)$ defined for $R_{0}<r<R$ and satisfying $0<\delta(r)<\pi$ such that

$$
f\left(r e^{i \theta}\right) \sim f(r) e^{i \theta a(r)-(1 / 2) \theta^{2} b(r)}
$$

as $r \rightarrow R$ uniformly for $|\theta| \leqq \delta(r)$; while uniformly for $\delta(r) \leqq|\theta| \leqq \pi$,

$$
f\left(r e^{i \theta}\right)=O\left(\frac{f(r)}{\sqrt{b(r)}}\right) \text { as } r \rightarrow R .
$$

THeOREM 3. (i) For admissible functions the positive real axis $i s$ an essential $\mu$ asymptotic path with $\mu$ asymptotic value $\infty$. Any path in an angle outside the positive real axis is a nonessential $\mu$ asymptotic path with $\mu$ asymptotic value 0 .

(ii) Let $f(z)$ be an admissible entire function satisfying the condition

$$
a(r)-\nu(r)=O\left(\frac{b(r)}{\log b(r)}\right)^{1 / 2} \text { as } r \rightarrow \infty,
$$

and let $0<c<\infty$. Then $c$ is a $\mu$ asymptotic value of $f(z)$ along the curve whose equation in polar coordinates is $(r, \phi(r))$ where

$$
\phi(r)=\left\{b(r)^{-1} \log 2 \pi c^{-2} b(r)\right\}^{1 / 2} .
$$

(iii) Let $g(z)=f\left(e^{i \psi} z\right)$ where $0<\psi<2 \pi$ and $f(z)$ is an admissible entire function for which (2.3) is satisfied and $\nu(r)$ assumes every integer as a value. If $0<c<\infty$, then $c$ is a $m$ asymptotic value of $g(z)$ along the curve $(r, \phi(r)-\psi)$ but $g(z)$ has no $\mu$ asymptotic values other than 0 and $\infty$.

In $§ 7$ we give some examples of functions illustrating our theorems.

3. Lemma 1. (cf. [6], [7]). Let $1 \leqq r \leqq\left(\rho\left(n_{k+1}\right) / \rho\left(n_{k}\right)\right)$. Then

$$
\frac{M\left(\rho\left(n_{k}\right) r\right)}{\mu\left(\rho\left(n_{k}\right) r\right)} \geqq \frac{\pi}{4}\left(1+r^{n_{k-1}-n_{k}}\right) \text {. }
$$

Proof. Let $\mu\left(\rho\left(n_{k}\right)\right)=\left|a_{n_{k}}\right| \rho\left(n_{k}\right)^{n_{k}}=\left|a_{n_{k-1}}\right| \rho\left(n_{k}\right)^{n_{k-1}}$. Then

$$
\begin{aligned}
& a_{n_{k}}\left\{\rho\left(n_{k}\right) r e^{i \theta}\right\}^{n_{k}}+a_{n_{k-1}}\left\{\rho\left(n_{k}\right) r e^{i \theta}\right\}^{n_{k-1}} \\
& \quad=\frac{1}{2 \pi i} \int_{|\xi|=\rho\left(n_{k}\right) r} \frac{f(\xi)}{\xi}\left\{\left(\frac{\rho\left(n_{k}\right) r e^{i \theta}}{\xi}\right)^{n_{k}}+\left(\frac{\rho\left(n_{k}\right) r e^{i \theta}}{\xi}\right)^{n_{k-1}}\right\} d \xi .
\end{aligned}
$$

Hence

$$
\left|a_{n_{k}}\left\{\rho\left(n_{k}\right) r e^{i \theta}\right\}^{n_{k}}+a_{n_{k-1}}\left\{\rho\left(n_{k}\right) r e^{i \theta}\right\}^{n_{k-1}}\right|
$$




$$
\leqq \frac{M\left(\rho\left(n_{k}\right) r\right)}{2 \pi} \int_{0}^{2 \pi}\left|1+e^{i \phi}\right| d \phi=\frac{4 M\left(\rho\left(n_{k}\right) r\right)}{\pi} .
$$

Choose $\theta=-\left(n_{k}-n_{k-1}\right)^{-1}\left(\arg a_{n_{k}}-\arg a_{n_{k-1}}\right)$. Then

$$
\left.\mu(\rho)\left(n_{k}\right) r\right)\left(1+r^{n_{k-1}-n_{k}}\right) \leqq \frac{4 M\left(\rho\left(n_{k}\right) r\right)}{\pi} .
$$

and the lemma follows.

Lemma 2. (cf. [9; pp. 71, 83]). Let $K>1$ and $0<c<\infty$. If $f(z)$ is admissible we may assume that $\delta(r)$ satisfies

$$
\left\{\frac{\log 2 \pi c^{-2} b(r)}{b(r)}\right\}^{1 / 2} \leqq \delta(r) \leqq\left\{\frac{K \log b(r)}{b(r)}\right\}^{1 / 2}
$$

for $r(c)<r<R$.

Proof. The first inequality must always be satisfied. Indeed admissibility implies

$$
\frac{\left|f\left(r e^{i \delta(r)}\right)\right|}{f(r)} \sim \exp \left\{-\frac{1}{2} b(r) \delta(r)^{2}\right\}=O\left(b(r)^{-1 / 2}\right) .
$$

Hence $\exp \left\{1 / 2 b(r) \delta(r)^{2}\right\} \geqq c^{-1}(2 \pi b(r))^{1 / 2}$ for $r(c)<r<R$, and this is equivalent to the first inequality.

For the second inequality suppose $f(z)$ is admissible with a function $\delta_{1}(r)$. Let $\delta(r)=\min \left\{\delta_{1}(r),(K \log b(r) / b(r))^{1 / 2}\right\}$. We show that $f(z)$ is admissible with $\delta(r)$. Let $\delta(r) \leqq|\theta| \leqq \delta_{1}(r)$. Then by (2.1)

$$
\frac{b(r)^{1 / 2}\left|f\left(r e^{i \theta}\right)\right|}{f(r)} \sim b(r)^{1 / 2} \exp \left\{-\frac{1}{2} b(r) \theta^{2}\right\} \leqq b(r)^{1 / 2-K / 2}=O(1) .
$$

This is equivalent to (2.2). Thus we may replace $\delta_{1}(r)$ by $\delta(r)$ without destroying the truth of (2.2).

4. Proof of Theorem 1. Without loss of generality, we may assume $f(0)=1$. Let $1<\alpha<\beta<L_{1}<L$, and $\alpha<(\pi /(4-\pi))^{1 / S}$. There exists a sequence $\left\{k_{p}\right\}=\{k(p)\}$ of integers such that $\rho\left(n_{k(p)}+1\right) / \rho\left(n_{k(p)}\right)>L_{1}$. Then if $\phi_{p}(w)=f\left(\rho\left(n_{k(p)}\right) w\right) / \mu\left(\rho\left(n_{k(p)}\right) w\right)$ for $w \in \Omega_{1}=\left\{w|1<| w \mid<L_{1}\right\}$, have, writing $n_{k_{p}}=n$, (cf. [6])

$$
|f(\rho(n) w)| \leqq 1+\sum_{k=1}^{\infty} \frac{\rho(n)^{k}|w|^{k}}{\rho(1) \cdots \rho(k)},
$$

and

$$
\mu(\rho(n)|w|)=\frac{\rho(n)^{n}|w|^{n}}{\rho(1) \cdots \rho(n)}
$$


Hence

$$
\begin{aligned}
\left|\phi_{p}(w)\right| & \leqq \frac{\rho(1) \cdots \rho(n)}{\rho(n)^{n}|w|^{n}}\left\{1+\sum_{k=1}^{\infty} \frac{\rho(n)^{k}|w|^{k}}{\rho(1) \cdots \rho(k)}\right\} \\
& =1+\sum_{j=1}^{\infty} \frac{\rho(n)^{j}|w|^{j}}{\rho(n+1) \cdots \rho(n+j)}+\sum_{j=-n}^{0} \frac{\rho(n+j+1) \cdots \rho(n)}{\rho(n)^{-j}|w|^{-j}} \\
& \leqq 1+\sum_{j=1}^{\infty}\left(\frac{|w|}{L_{1}}\right)^{j}+\sum_{j=1}^{\infty}|w|^{-j} \cdot
\end{aligned}
$$

Therefore $\left\{\phi_{p}(w)\right\}$ is uniformly bounded on compact subsets of $\Omega_{1}$ and so it is a normal family. Thus there is a subsequence of $\left\{\phi_{p}(w)\right\}$ which converges uniformly on compact subsets of $\Omega_{1}$. We may therefore assume that $\{k(p)\}$ has been so chosen that $\left\{\phi_{p}(w)\right\}$ itself converges uniformly on compact subsets of $\Omega_{1}$ to a holomorphic function $G(w)$.

We shall show that $G(w)$ is nonconstant, for suppose $G(w) \equiv C$ on $\Omega_{1}$ The constant term in the Laurent expansion of $\phi_{p}(w)$ about the origin is 1 , and so for $1<r<L_{1}$ we would have

$$
C=\frac{1}{2 \pi i} \int_{|w|=r} \frac{G(w)}{w} d w=\lim _{p \rightarrow \infty} \frac{1}{2 \pi i} \int_{|w|=r} \frac{\phi_{p}(w)}{w} d w=1 .
$$

Thus $G(w) \equiv 1$ on $\Omega_{1}$. But by the lemma

$$
M(r, G)=\lim _{p \rightarrow \infty} M\left(r, \phi_{p}\right) \geqq \frac{\pi}{4}\left(1+r^{-S}\right), \quad \text { for } 1<r<L_{1} .
$$

In particular for $r=\alpha$ we have $M(\alpha, G)>1$. Hence $G(w)$ must be nonconstant.

Let $\Omega=\{w|\alpha \leqq| w \mid \leqq \beta\}$ and suppose that $f(z)$ has a asymptotic value $\omega$. Then there exists a curve $\gamma(t)$ with $|\gamma(t)| \rightarrow \infty$ as $t \rightarrow \infty$ such that $f(\gamma(t)) / \mu(\gamma(t)) \rightarrow \omega$ as $t \rightarrow \infty$.

There exists an unbounded set $I$ with the following property: for each $t \in I$ there is a unique integer $p$ such that

$$
\rho\left(n_{k(p)}\right) \leqq|\gamma(t)|<\rho\left(n_{k(p)}+1\right) .
$$

Write $\gamma(t)=\rho\left(n_{k(p)}\right) \gamma_{p}(t)$; then $1 \leqq\left|\gamma_{p}(t)\right| \leqq L+o(1)$, so $\left\{\gamma_{p}(t)\right\}$ is bounded. We now consider the set $T$ of limit points of $\left\{\gamma_{p}(t)\right\}$ as $t \rightarrow \infty, t \in I$, which lie in $\Omega$ and prove they are an uncountable set on which $G(w)$ is constant. In fact, let $\Sigma$ be the intersection of $\Omega$ with the positive real axis, and define $\chi: \Sigma \rightarrow T$ as follows. For each $x \in \Sigma$, there exists $t_{p} \in I$ such that $\left|\gamma\left(t_{p}\right)\right|=\rho\left(n_{k(p)}\right) x$; then $\left|\gamma_{p}\left(t_{p}\right)\right|=x$. Choose a limit point $v$ of $\left\{\gamma_{p}\left(t_{p}\right)\right\}$, and define $\chi(x)=v$. Then $\chi$ is oneone since $|\chi(x)|=x$. Thus $T$ is uncountable, since $\Sigma$ is.

Furthermore $G(w)$ is constant on $T$, for suppose $\gamma_{p}\left(t_{s}\right) \rightarrow b \in T$ for a sequence $\left\{t_{s}\right\}$ with $t_{s} \in I$. By virtue of uniform convergence $\phi_{p}\left(\gamma_{p}\left(t_{s}\right)\right) \rightarrow G(b)$. But we are assuming $\omega$ is a $\mu$ asymptotic value and 
so $G(b)=\omega$. Hence $G$ is constant on $T$. This is a contradiction; therefore $G$ has no $\mu$ asymptotic values.

For $m$ asymptotic values we define

$$
\psi_{p}(w)=\frac{f\left(\rho\left(n_{k(p)}\right) w\right)}{m\left(\rho\left(n_{k(p)}\right) w\right)}
$$

for $w \in \Omega$,

and we still have (4.1) holding with $\phi_{p}$ replaced by $\psi_{p}$. Thus $\left\{\psi_{p}(w)\right\}$ is a normal family and the rest of the proof goes through in exactly the same manner as for $\mu$ asymptotic values.

5. Proof of theorem 2. Since $f(z)$ has positive coefficients we need only consider $\mu$ asymptotic values. We again suppose that $f(z)$ has $\mu$ asymptotic value $\omega$. Let $\gamma(t)$ be a $\mu$ asymptotic path corresponding to $\omega$. For a given $t$ take $m$ to be the unique integer for which $\rho\left(n_{m}\right) \leqq|\gamma(t)|<\rho\left(n_{m+1}\right)$ and define $\gamma_{m}(t)=C+i D$ where

$$
\gamma(t)=\rho\left(n_{m}\right) \exp \left(\frac{\phi C}{n_{m+1}-n_{m}}+i D\right)
$$

and $0 \leqq D<2 \pi$. It is easy to see that $0 \leqq R e \gamma_{m}(t) \leqq 1+o(1)$ so that $\left\{\gamma_{m}(t)\right\}$ is bounded.

Now write $P_{m}(w)=f(z) / \mu(z)$ where $z=\rho\left(n_{m}\right) \exp \left(\phi w /\left(n_{m+1}-n_{m}\right)\right)$. Then [8] $P_{m}(w)$ tends uniformly on $\Lambda=\{w \mid 0 \leqq R e w \leqq \beta\}, 1 / 2<\beta<1$, to a nonconstant analytic function $Q(w)$ as $m \rightarrow \infty$. For completeness we sketch a proof of this. We have

$$
1 \leqq \exp \left\{\frac{\phi R e w}{n_{m+1}-n_{m}}\right\}=\left|\exp \left(\frac{\phi w}{n_{m+1}-n_{m}}\right)\right| .
$$

For sufficiently large $m$

$$
0 \leqq \phi R e w \leqq \phi \beta<\left(n_{m+1}-n_{m}\right) \log \frac{\rho\left(n_{m+1}\right)}{\rho\left(n_{m}\right)},
$$

and so

$$
\rho\left(n_{m}\right) \leqq \rho\left(n_{m}\right)\left|\exp \left(\frac{\phi w}{n_{m+1}-n_{m}}\right)\right|=|z|<\rho\left(n_{m+1}\right)
$$

Hence

$$
\nu(|z|)=n_{m}, \mu(z)=\frac{z^{n_{m}}}{\rho(1) \cdots \rho\left(n_{m}\right)} .
$$

Write

$$
\sigma^{(j)}(m)= \begin{cases}\rho\left(n_{m+1}\right)^{n_{m+1}-n_{m}} \cdots \rho\left(n_{m+j}\right)^{n_{m+j}-n_{m+j-1}} & , \quad j>0 \\ 1 & , \quad j=0 \\ \left\{\rho\left(n_{m}\right)^{n_{m}-n_{m-1}} \cdots \rho\left(n_{m+j+1}\right)^{\left.n_{m+j+1}-n_{m+j}\right\}^{-1}},\right. & j<0 .\end{cases}
$$


Then

$$
\frac{f(z)}{\mu(z)}=\sum_{j=-m}^{\infty} \frac{\rho\left(n_{m}\right)^{n_{m+j}-n_{m}}}{\sigma^{(j)}(m)} \exp \left\{\frac{n_{m+j}-n_{m}}{n_{m+1}-n_{m}} \phi w\right\} .
$$

Since $1 \leqq a \leqq A<\infty$, and $\phi>0$, there exist numbers $A_{1}, A_{2}, \phi_{1}$ so that

$$
\begin{aligned}
& 0<A_{1}<\frac{n_{m+1}-n_{m}}{n_{m}-n_{m-1}}<A_{2}<\infty, \\
& 0<\phi_{1}<\left(n_{m+1}-n_{m}\right) \log \frac{\rho\left(n_{m+1}\right)}{\rho\left(n_{m}\right)}
\end{aligned}
$$$$
\text { for } m=1,2, \cdots \text {. }
$$

Let $j \geqq 2$. Then

$$
\begin{aligned}
\left(n_{m+j}-n_{m}\right) \log \rho\left(n_{m+1}\right)-\log \sigma^{(j)}(m) \\
\quad=-\sum_{q=2}^{j}\left(n_{m+j}-n_{m+q-1}\right) \log \frac{\rho\left(n_{m+q}\right)}{\rho\left(n_{m+q-1}\right)} \\
\leqq-\sum_{q=2}^{j}\left(n_{m+q}-n_{m+q-1}\right) \log \frac{\rho\left(n_{m+q}\right)}{\rho\left(n_{m+q-1}\right)} \\
\quad \leqq-(j-1) \phi_{1} .
\end{aligned}
$$

Similarly we have for $-j=k \geqq 2,\left(n_{m+j}-n_{m}\right) \log \rho\left(n_{m}\right)-\log \sigma^{(j)}(m) \leqq$ $(j+1) \phi_{1} / A_{2}$. Hence

$$
\left|\frac{z^{n_{m+j}-n_{m}}}{\sigma^{(j)}(m)}\right| \leqq\left\{\begin{array}{lr}
e^{-(j-1) \phi_{1}}, & j \geqq 2 \\
1 & , \quad-2<j<2 \\
e^{(j+1) \phi_{1} / A_{2}}, & j \leqq-2
\end{array}\right.
$$

Hence by the Weierstrass $M$-test the series (5.1) converges uniformly in both $m$ and $w$. Hence we have

$$
\lim _{m \rightarrow \infty} \frac{f(z)}{\mu(z)}=\sum_{-\infty}^{\infty} \lim _{m \rightarrow \infty}\left\{\frac{\rho\left(n_{m}\right)^{n_{m+j}-n_{m}}}{\sigma^{(j)}(m)} \exp \left(\frac{n_{m+j}-n_{m}}{n_{m+1}-n_{m}} \phi w\right)\right\} .
$$

Further for $j>0$

$$
\begin{aligned}
\left(n_{m+j}\right. & \left.-n_{m}\right) \log \rho\left(n_{m}\right)-\log \sigma^{(j)}(m) \\
& =-\sum_{q=1}^{j} \sum_{p=q}^{j}\left(\prod_{s=q}^{p-1} \frac{n_{m+s+1}-n_{m+s}}{n_{m+s}-n_{m+s-1}} \log \left(\frac{\rho\left(n_{m+q}\right)}{\rho\left(n_{m}\right)}\right)^{n_{m+q}-n_{m+q-1}}\right)
\end{aligned}
$$

and so

$$
\begin{aligned}
& \lim _{m \rightarrow \infty}\left\{\left(n_{m+j}-n_{m}\right) \log \rho\left(n_{m}\right)-\log \sigma^{(j)}(m)\right. \\
& \quad= \begin{cases}-\frac{1}{2} j(j+1) \phi & \text { if } A=a=1 \\
-\frac{\left(a^{j+1}-a(j+1)+j\right) \phi}{(a-1)^{2}} & \text { if } 1<a=A<\infty .\end{cases}
\end{aligned}
$$


A similar argument shows that (5.3) is valid when $j<0$. Hence we have from (5.2)

$$
\lim _{m \rightarrow \infty} \frac{f(z)}{\mu(z)}=\left\{\begin{array}{r}
\sum_{-\infty}^{\infty} \exp \left\{-\frac{\phi j}{2}(j+1-2 w)\right\} \text { when } A=a=1, \\
\sum_{-\infty}^{\infty} \exp \left\{-\frac{\phi}{(a-1)^{2}}\left(a^{j+1}-(j+1) a+j-(a-1)\left(a^{j}-1\right) w\right)\right\}, \\
\text { when } A=a>1 .
\end{array}\right.
$$

It can be easily verified that the two expressions on the right of (5.4) are not constant.

Just as in Theorem 1, we now can prove that the set $T$ of limit points of $\left\{\gamma_{m}(t)\right\}$ is uncountable, and that $Q(w)$ is constant on $T$, contradicting the fact that $Q(w)$ is nonconstant on $\Lambda$. Hence $f(z)$ has no $\mu$ asymptotic values.

6. Proof of Theorem 3. (i) We may assume by Lemma 2 that $\delta(r)=o(1)$. Furthermore according to [9] $a_{n}>0$ for $n>n_{0}$, and so we need only consider $\mu$ asymptotic values. We have [9; pp. 68-69]

$$
\frac{f\left(r e^{i \theta}\right)}{\mu\left(r e^{i \theta}\right)} \sim \sqrt{2 \pi b(r)} \exp \left\{i(a(r)-\nu(r)) \theta-\frac{1}{2} \theta^{2} b(r)\right\}
$$

uniformly for $|\theta| \leqq \delta(r)$, and

$$
\frac{f\left(r e^{i \theta}\right)}{\mu\left(r e^{i \theta}\right)}=o(1) \text { uniformly for } \delta(r) \leqq|\theta| \leqq \pi .
$$

It is immediate from (6.2) that any path in an angle outside the real axis has $\mu$ asymptotic value 0 and is nonessential. From (6.1) we have

$$
\frac{f(r)}{\mu(r)} \sim \sqrt{2 \pi b(r)},
$$

and so the positive real axis has $\mu$ asymptotic value $\infty$. To prove that it is essential it suffices to show that there exists a curve $(r, \phi(r))$ (in polar coordinates) such that for each $\varepsilon>0$ there exists $r(\varepsilon)$ for which $r>r(\varepsilon)$ implies $|\phi(r)|<\varepsilon$, and $(r, \phi(r))$ does not have $\mu$ asymptotic value $\infty$. We take

$$
\phi(r)=\left\{b(r)^{-1} \log \left(2 \pi c^{-2} b(r)\right)\right\}^{1 / 2},
$$

where $0<c<\infty$. Then by Lemma $2,|\phi(r)| \leqq \delta(r)$ for $r>r(c)$ and

$$
\left|\frac{f\left(r e^{i \phi(r)}\right)}{\mu\left(r e^{i \phi(r)}\right)}\right| \sim \sqrt{2 \pi b(r)} e^{-(1 / 2) \phi(r)^{2} b(r)}=c,
$$


so that $(r, \phi(r))$ cannot have $\mu$ asymptotic value $\infty$.

(ii) If (2.3) is satisfied for $f(z)$ then $(a(r)-\nu(r)) \phi(r)=o(1)$, and so

$$
\frac{f\left(r e^{i \phi(r)}\right)}{\mu\left(r e^{i \phi(r)}\right)} \sim \sqrt{2 \pi b(r)} e^{-(1 / 2) \phi(r)^{2} b(r)}=c .
$$

(iii) We have

$$
\frac{g\left(r e^{i(\phi(r)-\psi)}\right)}{m\left(r e^{i(\phi(r)-\psi)}, g\right)}=\frac{f\left(r e^{i \phi(r)}\right)}{\mu\left(r e^{i \phi(r)}, f\right)} \sim c,
$$

so that $c$ is a $m$ asymptotic value of $g(z)$ along $(r, \phi(r)-\psi)$. However

$$
\frac{g\left(r e^{i(\theta-\psi)}\right)}{\mu\left(r e^{i(\theta-\psi r)}, g\right)} \sim e^{i \psi \nu \nu(r)} \sqrt{2 \pi b(r)} e^{i(a(r)-\nu(r)) \theta-1 / 2 b(r) \theta^{2}}
$$

uniformly for $0 \leqq|\theta| \leqq \delta(r)$. Since $\nu(r)$ assumes every integer as a value, $g$ can have no $\mu$ asymptotic values other than 0 and $\infty$.

7. Examples. (i) Theorem 1 shows that $\sum_{n=0}^{\infty} \lambda^{-(1 / 2) n(n+1)} e^{i \alpha} z^{n}$, where $1<\lambda<\infty$ and $0 \leqq \alpha_{n}<2 \pi$, has no $\mu$ or $m$ asymptotic values. Here $\rho(n)=\lambda^{n}$ and $L=\lambda$. Similarly it follows from Theorem 2 that if $0<\alpha<\infty$ the functions

$$
\sum_{k=0}^{\infty} \frac{z^{k^{2}}}{\left(k^{2} !\right)^{\alpha}}, \sum_{k=0}^{\infty} \frac{z^{k^{2}}}{\Gamma\left(1+\alpha k^{2}\right)},
$$

and $\sum_{k=0}^{\infty} z^{k^{2}} / k^{2 \alpha k^{2}}$ have no $\mu$ or $m$ asymptotic values. For each of these functions $\phi=4 \alpha$.

(ii) The function $e^{z}$ is admissible with $a(r)=b(r)=r$ and $\nu(r)=$ $[r]$, so Theorem 3 (i), (ii) apply to it. More generally the MittagLeffler function

$$
E_{\alpha}(z)=\sum_{n=0}^{\infty} \frac{z^{n}}{\Gamma(1+\alpha n)}(0<\alpha<2)
$$

is admissible with $a(r)=\alpha^{-1} r^{a^{-1}}+o(1), b(r)=\alpha^{-2} r^{\alpha^{-1}}+o(1)$, and $\nu(r)=$ $\alpha^{-1} r^{a^{-1}}+O(1)$, so that $a(r)-\nu(r)=O(1)$. These facts follow from

$$
E_{\alpha}(z)-e^{z^{1 / \alpha}}=O(1 / z) \text { for }|\arg z| \leqq \frac{1}{2} \alpha \pi \quad[1 ; \text { p. 175] }
$$

If $f(z)$ is admissible so is $e^{f(z)}$ [9].

(iii) Let $L_{\beta}(z)=\sum_{n=0}^{\infty}\{z /(\log (n+\beta))\}^{n}$ where $\beta>1$. It is known [3; p. 346] that $L_{\beta}(z)$ tends to zero on every ray except the real axis, where it tends to $\infty$. Hence $e^{-L_{\beta}(z)}$ has $\mu$ and $m$ asymptotic value 0 along every ray from 0 to $\infty$.

(iv) Theorem 3 (ii), (iii) show that every positive real number is 
an $m$ asymptotic value of some function. If $b$ is any complex number we can construct a function having $b$ as a $\mu$ asymptotic value. We take for example

$$
f(z, b)=e^{i z^{2}}+\frac{b}{z \sqrt{2 \pi}}\left(e^{z^{2}}-1\right) .
$$

For $r>(|b|) /(\sqrt{2 \pi})$ we have $\mu(r, f)=\mu\left(r, e^{i z^{2}}\right) \sim\left(e^{r^{2}}\right) /(r \sqrt{2 \pi})$. It follows easily that $\lim _{r \rightarrow \infty} f(r) / \mu(r)=b$.

We wish to thank the referee for his suggestions.

Added in proof. An application of these results may be found in the authors' paper "Asymptotic values of holomorphic functions of irregular growth," Bull. Amer. Math. Soc. 71 (1965), 747-749.

\section{REFERENCES}

1. L. Bieberbach, Lehrbuch der Funktionentheorie (II), New York, 1945.

2. J. Clunie and W. K. Hayman, The maximum term of a power series, J. d'Analyse Math. 12 (1964), 143-186.

3. P. Dienes, The Taylor series, New York, 1957.

4. P. Erdös, Some unsolved problems, Michigan Math. J. 4 (1957), 291-300.

5. P. Erdös and A. J. Macintyre, Integral functions with gap power series, Edinburgh Math. Proc. (2) 10 (1954), 62-70.

6. Alfred Gray and S.M. Shah, A note on entire functions and a conjecture of Erdös, Bull. Amer. Math. Soc. 69 (1963), 573-577.

7. — A note on entire functions and a conjecture of Erdös (II), J. d'Analyse Math. 12 (1964), 83-104.

8. - Holomorphic functions with gap power series, Math. Zeit. 86 (1965), 375394.

9. W. K. Hayman, A generalization of Stirling's formula, J. Reine Angew. Math. 196 (1956), 67-95.

10. S. M. Shah, The behavior of entire functions and a conjecture of Erdös, Amer. Math. Monthly 68 (1961), 419-425.

Received August 5, 1964, and in revised form December 30, 1964. This work was supported by NSF Grants GP-209 and GP-2572.

University of CALIFornia, Berkeley

UNIVERSITY OF KANSAS, LAWRENCE 


\section{PACIFIC JOURNAL OF MATHEMATICS}

H. SAMELSON

Stanford University Stanford, California

R. M. BLUMENTHAL

University of Washington

Seattle, Washington 98105

\section{EDITORS}

\author{
*J. DugundJI \\ University of Southern California \\ Los Angeles, California 90007 \\ RICHARD ARENS \\ University of California \\ Los Angeles, California 90024
}

\section{ASSOCIATE EDITORS}

E. F. BeCKENBACH

B. H. NEUMANN

F. WOLF

K. YosidA

\section{SUPPORTING INSTITUTIONS}

UNIVERSITY OF BRITISH COLUMBIA

CALIFORNIA INSTITUTE OF TECHNOLOGY

UNIVERSITY OF CALIFORNIA

MONTANA STATE UNIVERSITY

UNIVERSITY OF NEVADA

NEW MEXICO STATE UNIVERSITY

OREGON STATE UNIVERSITY

UNIVERSITY OF OREGON

OSAKA UNIVERSITY

UNIVERSITY OF SOUTHERN CALIFORNIA
STANFORD UNIVERSITY

UNIVERSITY OF TOKYO

UNIVERSITY OF UTAH

WASHINGTON STATE UNIVERSITY

UNIVERSITY OF WASHINGTON

AMERICAN MATHEMATICAL SOCIETY CHEVRON RESEARCH CORPORATION TRW SYSTEMS

NAVAL ORDNANCE TEST STATION 


\section{Pacific Journal of Mathematics}

Vol. 18, No. 1

March, 1966

Edward Joseph Barbeau, Semi-algebras that are lower semi-lattices ...... 1

Steven Fredrick Bauman, The Klein group as an automorphism group

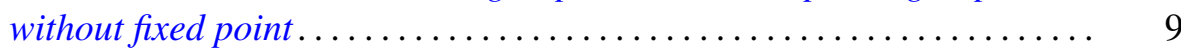

Homer Franklin Bechtell, Jr., Frattini subgroups and $\Phi$-central groups .... 15

Edward Kenneth Blum, A convergent gradient procedure in prehilbert

spaces ............................................

Edward Martin Bolger, The sum of two independent exponential-type random variables ...................................

David Wilson Bressler and A. P. Morse, Images of measurable sets .......

Dennison Robert Brown and J. G. LaTorre, A characterization of uniquely

divisible commutative semigroups........................

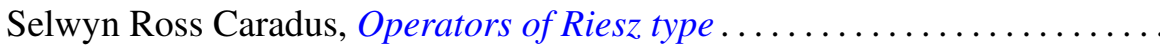

Jeffrey Davis and Isidore Isaac Hirschman, Jr., Toeplitz forms and ultraspherical polynomials ............................

Lorraine L. Foster, On the characteristic roots of the product of certain rational integral matrices of order two ......................

Alfred Gray and S. M. Shah, Asymptotic values of a holomorphic function

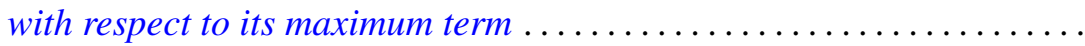

Sidney (Denny) L. Gulick, Commutativity and ideals in the biduals of

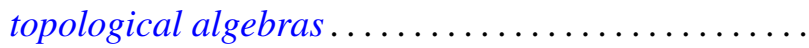

G. J. Kurowski, Further results in the theory of monodiffric functions

Lawrence S. Levy, Commutative rings whose homomorphic images are self-injective .

Calvin T. Long, On real numbers having normality of order $k$....

Bertram Mond, An inequality for operators in a Hilbert space. ...

John William Neuberger, The lack of self-adjointness in three-point boundary value problems ........................

C. A. Persinger, Subsets of $n$-books in $E^{3}$

Oscar S. Rothaus and John Griggs Thompson, A combinatorial problem in the symmetric group ............................... 175

Rodolfo DeSapio, Unknotting spheres via Smale .................. 179

James E. Shockley, On the functional equation

$$
F(m n) F((m, n))=F(m) F(n) f((m, n)) \ldots \ldots \ldots
$$

Kenneth Edward Whipple, Cauchy sequences in Moore spaces ... 